\title{
An acoustic eikonal equation for attenuating transversely isotropic media with a vertical symmetry axis
}

\author{
Qi $\mathrm{Hao}^{1}$ and Tariq Alkhalifah ${ }^{2}$
}

\begin{abstract}
Seismic-wave attenuation is an important component of describing wave propagation. Certain regions, such as gas clouds inside the earth, exert highly localized attenuation. In fact, the anisotropic nature of the earth induces anisotropic attenuation because the quasi P-wave dispersion effect should be profound along the symmetry direction. We have developed a $2 \mathrm{D}$ acoustic eikonal equation governing the complex-valued traveltime of quasi $\mathrm{P}$-waves in attenuating, transversely isotropic media with a vertical-symmetry axis (VTI). This equation is derived under the assumption that the complex-valued traveltime of quasi P-waves in attenuating VTI media are independent of the S-wave velocity parameter $v_{\mathrm{S} 0}$ in Thomsen's notation and the S-wave attenuation coefficient $A_{\mathrm{S} 0}$ in Zhu and Tsvankin's notation. We combine perturbation theory and Shanks transform to develop practical approximations to the acoustic attenuating eikonal equation, capable of admitting an analytical description of the attenuation in homogeneous media. For a horizontal-attenuating VTI layer, we also
\end{abstract}

derive the nonhyperbolic approximations for the real and imaginary parts of the complex-valued reflection traveltime. These equations reveal that (1) the quasi SV-wave velocity and the corresponding quasi $\mathrm{SV}$-wave attenuation coefficient given as part of Thomsen-type notation barely affect the ray velocity and ray attenuation of quasi P-waves in attenuating VTI media; (2) combining the perturbation method and Shanks transform provides an accurate analytic eikonal solution for homogeneous attenuating VTI media; (3) for a horizontal attenuating VTI layer with weak attenuation, the real part of the complex-valued reflection traveltime may still be described by the existing nonhyperbolic approximations developed for nonattenuating VTI media, and the imaginary part of the complex-valued reflection traveltime still has the shape of nonhyperbolic curves. In addition, we have evaluated the possible extension of the proposed eikonal equation to realistic attenuating media, an alternative perturbation solution to the proposed eikonal equation, and the feasibility of applying the proposed nonhyperbolic equation for the imaginary part of the complex-valued traveltime to invert for interval attenuation parameters.

\section{INTRODUCTION}

Modeling the anelastic attenuating nature of the earth is becoming important because our analysis of recorded data includes a closer look at amplitude for inversion purposes. The anisotropic attenuation of rocks is found from ultrasonic observation in the laboratory (Tao and King, 1990; Best et al., 2007; Zhubayev et al., 2016) and from a field seismic survey (Carter and Kendall, 2006; Clark et al., 2009; Behura et al., 2012; Shekar and Tsvankin, 2012). Because waves tend to exhibit anisotropic behavior as a result of the natural thin layering of the earth, we would expect the same waves to experience an anisotropic attenuation for the same reason.
Describing such behavior in an efficient manner via complex traveltimes, and accordingly acquiring insights of its influence on seismic data are important for proper wave propagation description and inversion. The theory of wave propagation in attenuating media is systematically presented in Borcherdt (2009) and Carcione (2015, pp. 63-229).

The traveltime of body waves in an attenuating medium is complex valued. The real part of the complex-valued traveltime corresponds to the phase of the waves, whereas the imaginary part affects the decay of the amplitude of the waves due to energy absorption. The complex-valued traveltime of body waves in attenuating media satisfies the complex eikonal equation (Červený and Pšenčík, 2009).

\footnotetext{
Manuscript received by the Editor 28 March 2016; revised manuscript received 2 August 2016; published online 21 November 2016.

${ }^{1}$ Norwegian University of Science and Technology (NTNU), Department of Petroleum Engineering and Applied Geophysics, Trondheim, Norway. E-mail: qi. hao@ntnu.no.

${ }^{2}$ King Abdullah University for Science and Technology (KAUST), Physical Science Division, Thuwal, Saudi Arabia. E-mail: tariq.alkhalifah@kaust.edu.sa.

(C) 2017 Society of Exploration Geophysicists. All rights reserved.
} 
As a fundamental equation, the eikonal equation plays an important role in the forward modeling and inversion of traveltime in attenuating media.

Similar to the real ray-tracing method developed for elastic anisotropic media, the complex ray tracing theory has been developed to solve the complex-valued eikonal equation (Zhu and Chun, 1994; Thomson, 1997; Chapman et al., 1999; Kravtsov et al., 1999; Hanyga and Seredyňska, 2000; Amodei et al., 2006). The complex ray theory is very accurate in solving the complex eikonal equation, and it is applicable to anisotropy and attenuation of arbitrary strength (Vavryčuk, 2010). Because the trajectory of a complex ray is defined in complex space, the complex ray-tracing method requires that the model parameters have to be extended into complex space, which cannot be accomplished for realistic 3D velocity models (Vavryčuk, 2012). As a result, it is difficult to implement complex ray tracing in practice. For horizontally layered, attenuating media, the traveltime of complex rays may be calculated by determining the horizontal slowness component at the saddle point of the phase function of the wavefield at the receiver (Hearn and Krebes, 1990a, 1990b; Krebes and Slawinski, 1991; Le et al., 1994). By assuming that wave attenuation is weak and considering the imaginary parts of complex-valued stiffness coefficients as the perturbation parameters, perturbation approaches have been developed to approximately calculate the complex-valued traveltime of body waves in attenuating media (Gajewski and Pšenčík, 1992; Červený [2001], pp. 542-548; Červený and Pšenčík, 2009; Klimeš and Klimeš, 2011). In perturbation approaches, the complex-valued traveltime is expressed in terms of the perturbation of medium parameters from the nonattenuating case to the attenuating case, and the complex-valued traveltime is calculated along the real raypath in the nonattenuating reference medium. Therefore, the perturbation approaches are simple and fast compared with complex ray tracing. Despite being less accurate for strongly attenuating media, perturbation approaches are applicable to realistic models. As an alternative, Vavryčuk (2008, 2010,2012 ) proposes a real ray-tracing method to solve the complex eikonal equation for general attenuating anisotropic media. In this method, the ray-tracing equations are reconstructed such that the raypath is entirely located in real space. Because this method needs neither a real reference medium nor the continuation of medium parameters defined in real space to complex space, it is accurate and stable for strongly attenuating media.

In addition to ray-tracing methods, numerical techniques involved in directly solving the eikonal equation after spatial discretization, such as finite-difference (Vidale, 1988, 1990; van Trier and Symes, 1991), fast-sweeping (Zhang et al., 2006; Luo and Qian, 2012), and fast-marching (Sethian, 1996; Sethian and Vladimirsky, 2001; Alkhalifah, 2011) methods, have been widely used in calculating traveltime for nonattenuating media. However, these methods cannot be applied to attenuating media because they need to update the traveltime in the grids along the direction of wavefront expansion by selecting the minimum of traveltimes in a heap, but selecting the minimum traveltime is not valid for complex-valued traveltimes in attenuating media. Until now, the numerical eikonal solutions have been limited to nonattenuating media.

The main goal of this paper is to develop an acoustic eikonal equation for attenuating transversely isotropic media with a vertical symmetry axis (VTI), in which the symmetry of the attenuation of waves is the same as that of the phase velocity. Zhu and Tsvankin (2006) propose a Thomsen-type notation (see equations A-6-A-10 in Appendix A) to describe the anisotropy of attenuation coefficient for homogeneous plane waves in attenuating VTI media. They find that the attenuation coefficient of the homogeneous plane quasi $\mathrm{P}$-wave is almost independent of the $\mathrm{S}$-wave attenuation coefficient $A_{\mathrm{S} 0}$ in the Thomsen-type notation. For nonattenuating VTI media, the influence of the parameter $v_{\mathrm{S} 0}$ in Thomsen's (1986) notation on the traveltime and velocity of quasi P-waves is very weak and generally negligible (Alkhalifah, 1998, 2000). Alkhalifah (1998, 2000, 2003 ) proposes the acoustic approximation and derives the acoustic eikonal and wave equations for nonattenuating VTI and orthorhombic media. Besides Voigt notation and Zhu and Tsvankin's (2006) notation, it is worth mentioning that two different weak anisotropyattenuation notations are proposed by Vavryčuk (2009) and Rasolofosaon (2010) to describe the attenuation of waves in general attenuating anisotropic media.

We will combine Thomsen's (1986) notation and Zhu and Tsvankin's (2006) notation to fully parameterize an attenuating VTI medium (see Appendix A). We will use the acoustic approximation to derive an acoustic eikonal equation for an attenuating VTI medium. Compared with the exact eikonal equation of quasi $\mathrm{P}$ - and SV-waves in an attenuating VTI medium, the acoustic eikonal equation includes fewer medium parameters because we will not consider the S-wave velocity parameter $v_{\mathrm{S} 0}$ in Thomsen's (1986) notation and the S-wave attenuation-coefficient parameter $A_{\mathrm{S} 0}$ in Zhu and Tsvankin's (2006) notation. Similar to the nonattenuating acoustic eikonal equations (Alkhalifah, 2000, 2003), the acoustic eikonal equation proposed will provide a choice of modeling and inversion of P-wave complex-valued traveltimes without preliminarily knowing the $\mathrm{S}$-wave parameters $v_{\mathrm{S} 0}$ and $A_{\mathrm{S} 0}$. However, we must emphasize that the acoustic eikonal equation is not the exact one that controls the quasi P-wave complex-valued traveltimes in an attenuating VTI medium, although the first example in the "Numerical examples" section will prove that the S-wave parameters $v_{\mathrm{S} 0}$ and $A_{\mathrm{S} 0}$ barely affect the velocity and attenuation of P-wave rays in a VTI model with strong attenuation anisotropy. The remainder of the paper is organized as follows: We start with the exact eikonal equation for attenuating VTI media. Then, we will consider the acoustic assumption to approximate the exact eikonal equation, from which we derive the acoustic eikonal equation for attenuating VTI media. Furthermore, we present a perturbation method to approximately calculate the complex-valued traveltime from the acoustic eikonal equation for a homogeneous, attenuating VTI medium. Shanks transform is implemented to accelerate the convergence of the traveltime series with respect to the perturbation parameters. This treatment to the acoustic eikonal equation, in nature, is almost the same as the method proposed by Alkhalifah (2011) and Stovas and Alkhalifah (2012) to solve the acoustic eikonal equations for nonattenuating anisotropic media. From the approximate solution to the acoustic attenuating eikonal equation, we also derive the approximations for the real and imaginary parts of the complex-valued traveltime of quasi P-waves in a horizontal, homogeneous, attenuating VTI layer.

\section{THE EXACT EIKONAL EQUATION}

According to the correspondence principle (Ben-Menahem and Singh [1981], pp. 875; Carcione [2015], pp. 145-146), attenuation is incorporated into the wavefield modeling by substituting the realvalued frequency-independent stiffness coefficients with the complex-valued frequency-dependent stiffness coefficients. For a general 
attenuating anisotropic medium, the frequency-domain stiffness coefficients $c_{i j}$ in Voigt notation are expressed by (Červený and Pšenčík, 2005, 2009)

$$
c_{i j}=c_{i j}^{R}-i c_{i j}^{I}
$$

where the symbol $i$ denotes the imaginary unit, $c_{i j}^{R}$ and $c_{i j}^{I}$ are real valued, and $c_{i j}^{I}$ becomes zero for nonattenuating media; the minus sign corresponds to the sign in the exponential factor $\exp (-i \omega t)$ of a time-harmonic wave under consideration (Červený and Pšenčík, 2009), where $\omega$ and $t$ denote the angular frequency and time. For a nonattenuating anisotropic medium, the matrix composed of $c_{i j}$ is positive definite, which is derived from the fact that strain energy is positive (Fedorov [1968], pp. 12-18; Carcione [2015], pp. 5-7). For a time-harmonic plane wave with the exponential factor $\exp (-i \omega t)$, the time-averaged strain energy and the dissipated energy are positive in an attenuating anisotropic medium. This requires that matrices composed of $c_{i j}^{R}$ and $c_{i j}^{I}$ are also positive definite (Červený and Pšenčík, 2006).

The zeroth-order time-harmonic ray solution to the elastodynamic equation for an attenuating medium, in accordance with equation 1 , is expressed by (Gajewski and Pšenčík, 1992; Vavryčuk, 2007, 2010)

$$
\mathbf{u}(\mathbf{x}, t)=\mathbf{U}(\mathbf{x}) \exp (-i \omega(t-\tau(\mathbf{x}))),
$$

where $\mathbf{x}$ denotes the position vector; $t$ denotes the time; $\omega$ denotes the angular frequency; $\mathbf{u}$ is the particle displacement vector; $\mathbf{U}(\mathbf{x})$ denotes the vector including factors - such as the spectrum of the source wavelet, radiation pattern, geometric spreading, reflection, and transmission - that affect the wave amplitude along the raypath; the direction of $\mathbf{U}(\mathbf{x})$ is identical to the polarization direction of particle displacement; and $\tau=\tau_{R}+i \tau_{I}$ denotes the complexvalued traveltime of rays.

The time-dependent exponential term in equation 2 is factorized into

$$
\exp (-i \omega(t-\tau(\mathbf{x})))=\exp \left(-i \omega\left(t-\tau_{R}(\mathbf{x})\right)\right) \exp \left(-\omega \tau_{I}(\mathbf{x})\right)
$$

Here, the factor $\exp \left(-\omega \tau_{I}(\mathbf{x})\right)$ describes the decay of the amplitude due to energy absorption. Therefore, $\tau_{I}$ must always be positive for positive angular frequencies, which corresponds to the stiffness coefficients defined in equation 1 , and it must be negative for negative angular frequencies, which corresponds to the stiffness coefficients described by the complex conjugate of equation 1 . In the case of attenuating isotropic media, this requirement is identical to the one that the quality factors of quasi $\mathrm{P}$ - and S-waves are odd functions of frequency (Aki and Richards [2002], pp. 163-165).

Substitution of equation 2 into the elastodynamic equation for a generally attenuating anisotropic medium leads to the Christoffel equation (Vavryčuk, 2007). The Christoffel equation of quasi Pand $\mathrm{SV}$-waves in the $[x, z]$ plane of an attenuating VTI medium is given by

$$
\left(\begin{array}{cc}
a_{11} p_{1}^{2}+a_{55} p_{3}^{2}-1 & \left(a_{13}+a_{55}\right) p_{1} p_{3} \\
\left(a_{13}+a_{55}\right) p_{1} p_{3} & a_{55} p_{1}^{2}+a_{33} p_{3}^{2}-1
\end{array}\right)\left(\begin{array}{l}
g_{1} \\
g_{3}
\end{array}\right)=0,
$$

where $a_{i j}=c_{i j} / \rho$ denote the density-normalized stiffness coefficients, where $c_{i j}$ is defined in equation 1 ; for an attenuating VTI medium, the density-normalized stiffness coefficients $a_{i j}$ are de- scribed by the combination of Thomsen's (1986) notation and Zhu and Tsvankin's (2006) notation (see Appendix A); $p_{1}$ and $p_{3}$ denote the horizontal and vertical slowness components, respectively; $g_{1}$ and $g_{3}$ denote the horizontal and vertical polarization components, respectively.

In the time-space domain, the phase slowness is expressed by the spatial derivative of traveltime:

$$
p_{1}=\frac{\partial \tau}{\partial x}, \quad p_{3}=\frac{\partial \tau}{\partial z}
$$

From equations 4 and 5, we obtain the 2D eikonal equation for attenuating VTI media,

$$
\begin{aligned}
& \left(a_{11}\left(\frac{\partial \tau}{\partial x}\right)^{2}+a_{55}\left(\frac{\partial \tau}{\partial z}\right)^{2}-1\right)\left(a_{55}\left(\frac{\partial \tau}{\partial x}\right)^{2}+a_{33}\left(\frac{\partial \tau}{\partial z}\right)^{2}-1\right) \\
& -\left(a_{13}+a_{55}\right)^{2}\left(\frac{\partial \tau}{\partial x}\right)^{2}\left(\frac{\partial \tau}{\partial z}\right)^{2}=0 .
\end{aligned}
$$

This eikonal equation governs the P- and SV-wave traveltimes and has the same form as in nonattenuating media. The 3D eikonal equation may be easily obtained by replacing the square of the partial derivative of $\tau$ with respect to $x$-axis by a sum of the square of the partial derivatives of $\tau$ with respect to the $x$ - and $y$-axes.

\section{AN ACOUSTIC ATTENUATING EIKONAL EQUATION}

The parameter $v_{\mathrm{S} 0}$ in Thomsen's (1986) notation barely affects the quasi P-wave velocity in elastic VTI media, which is called the acoustic approximation (Alkhalifah, 1998, 2000). For attenuating anisotropic media, the attenuation coefficient of plane quasi $\mathrm{P}$ waves is approximately independent of the $\mathrm{S}$-wave attenuation coefficient $A_{\mathrm{S} 0}$ (Zhu and Tsvankin, 2006). Therefore, setting $v_{\mathrm{S} 0}=$ 0 and $A_{\mathrm{S} 0}=0$ should have little influence on quasi P-wave complex-valued traveltime in an attenuating VTI medium described by the combination of Thomsen's (1986) and Zhu and Tsvankin's (2006) notations, and it will lead to the acoustic attenuating VTI medium. In fact, we only need to consider the acoustic approximation $\left(v_{\mathrm{S} 0}=0\right)$ to obtain the acoustic attenuating eikonal equation, without any assumption on $A_{\mathrm{S} 0}$. This is because $A_{\mathrm{S} 0}$ appears with $v_{\mathrm{S} 0}$ in the form $A_{\mathrm{S} 0} v_{\mathrm{S} 0} /\left(1-A_{\mathrm{S} 0}^{2}\right)$, when expressing the stiffness coefficients in terms of parameters in Thomsen's (1986) and Zhu and Tsvankin's (2006) notations. In this case, we further replace Thomsen's (1986) notation by Alkhalifah's $(1998,2000)$ notation to describe an acoustic nonattenuating VTI reference because Alkhalifah's (1998, 2000) notation is more closely linked to the P-wave velocity anisotropy. Alkhalifah's $(1998,2000)$ notation includes three parameters: the P-wave vertical phase velocity $v_{\mathrm{P} 0}$, the quasi P-wave normal-moveout (NMO) velocity $v_{n}=v_{\mathrm{P} 0} \sqrt{1+2 \delta}$, and the anellipticity parameter $\eta=(\varepsilon-\delta) /(1+2 \delta)$, where $\varepsilon$ and $\delta$ are Thomsen (1986) anisotropy parameters (see equations A-3 and A-4 in Appendix A). The attenuation part of an acoustic attenuating VTI medium is characterized by the following parameters in Zhu and Tsvankin's (2006) notation: the P-wave vertical attenuation coefficient $A_{\mathrm{P} 0}$, the fractional difference $\varepsilon_{Q}$ between the horizontal and vertical attenuation coefficients, and the second-order derivative $\delta_{Q}$ of attenuation coefficient with respect to the phase angle of homogeneous plane quasi $\mathrm{P}$-waves along the vertical direction 
(see equations A-6, A-8, and A-9 in Appendix A), where the phase angle is measured from the phase propagation direction to the vertical direction.

It follows that the 2D acoustic attenuating VTI eikonal equation is given by

$$
A\left(\frac{\partial \tau}{\partial x}\right)^{2}+B\left(\frac{\partial \tau}{\partial z}\right)^{2}+C\left(\frac{\partial \tau}{\partial x}\right)^{2}\left(\frac{\partial \tau}{\partial z}\right)^{2}=1,
$$

where coefficients $A, B$, and $C$ are given by

$$
\begin{gathered}
A=v_{n}^{2}(1+2 \eta)\left(1-2 i k\left(1+\varepsilon_{Q}\right)\right), \\
B=v_{\mathrm{P} 0}^{2}(1-2 i k), \\
C=\frac{v_{\mathrm{P} 0}^{2}}{v_{n}^{2}}\left((1-2 i k) v_{n}^{2}-i k \delta_{Q} v_{\mathrm{P} 0}^{2}\right)^{2} \\
-v_{\mathrm{P} 0}^{2} v_{n}^{2}(1+2 \eta)(1-2 i k)\left(1-2 i k\left(1+\varepsilon_{Q}\right)\right),
\end{gathered}
$$

with

$$
k=\frac{A_{\mathrm{P} 0}}{1-A_{\mathrm{P} 0}^{2}} .
$$

The acoustic eikonal equation 7 implies that the complex-valued traveltime of quasi P-waves in an attenuating VTI medium under acoustic approximation is independent of S-wave parameters $v_{\mathrm{S} 0}$ and $A_{\mathrm{S} 0}$.

For weakly attenuating VTI media, we assume that the attenuation coefficient $A_{\mathrm{P} 0} \ll 1$. The linear approximations for equations 8-10 with respect to $A_{\mathrm{P} 0}$ are given by

$$
\begin{gathered}
A \approx v_{n}^{2}(1+2 \eta)\left(1-2 i A_{\mathrm{P} 0}\left(1+\varepsilon_{Q}\right)\right) \\
B \approx v_{\mathrm{P} 0}^{2}\left(1-2 i A_{\mathrm{P} 0}\right) \\
C \approx-2 v_{\mathrm{P} 0}^{2} v_{n}^{2} \eta+2 i A_{\mathrm{P} 0} v_{\mathrm{P} 0}^{2}\left(4 v_{n}^{2} \eta-v_{\mathrm{P} 0}^{2} \delta_{Q}+v_{n}^{2} \varepsilon_{Q}(1+2 \eta)\right) .
\end{gathered}
$$

Note that in the acoustic eikonal equation 7, the imaginary part of the traveltime vanishes when $A_{\mathrm{P} 0}=0$, implying no attenuation, and the traveltime is real valued. In this case, the acoustic eikonal equation fully reduces to the one for acoustic nonattenuating VTI media (Alkhalifah, 1998, 2000).

\section{THE ANALYTIC SOLUTION TO THE ACOUSTIC EIKONAL EQUATION FOR HOMOGENEOUS MEDIA}

To gain some insight into the acoustic eikonal equation 7 , let us consider the special case of a homogeneous, attenuating VTI medium to derive the approximate analytic solution. We assume that the source is located at the origin of a 2D Cartesian coordinate system for simplicity.

We define the vector $\ell=\left(\eta, \varepsilon_{Q}, \delta_{Q}\right)^{T}$ to represent the trial solution to equation 7 ,

$$
\tau=\tau_{0}+\sum_{i=1}^{3} \tau_{i} \ell_{i}+\sum_{i, j=1 ; i \leq j}^{3} \tau_{i j} \ell_{i} \ell_{j} .
$$

Equation 15 describes a second-order approximation for the complex-valued traveltime in terms of the anellipticity parameter and the Thomsen-type attenuation-anisotropy parameters because they are independent parameters and relatively small. Substitution of equation 15 into equation 7 with equations $8-11$ gives a secondorder expansion of the eikonal equation with respect to the anellipticity parameter and Thomsen-type attenuation-anisotropy parameters. Because all zeroth-, first-, and second-order coefficients in the expansion must equal zero to satisfy the eikonal equation, we derive the governing equations for the traveltime coefficients as follows:

- The zeroth-order traveltime coefficient $\tau_{0}$ satisfying

$$
v_{n}^{2}\left(\frac{\partial \tau_{0}}{\partial x}\right)^{2}+v_{\mathrm{P} 0}^{2}\left(\frac{\partial \tau_{0}}{\partial z}\right)^{2}=\frac{1-A_{\mathrm{P} 0}^{2}}{\left(1-i A_{\mathrm{P} 0}\right)^{2}} .
$$

- The first-order traveltime coefficients $\tau_{i}$ satisfying

$$
v_{n}^{2} \frac{\partial \tau_{0}}{\partial x} \frac{\partial \tau_{i}}{\partial x}+v_{P 0}^{2} \frac{\partial \tau_{0}}{\partial z} \frac{\partial \tau_{i}}{\partial z}=f_{i}\left(\tau_{0}\right), \quad i=1,2,3 .
$$

- The second-order traveltime coefficients $\tau_{i j}$ satisfying

$$
\begin{gathered}
v_{n}^{2} \frac{\partial \tau_{0}}{\partial x} \frac{\partial \tau_{i j}}{\partial x}+v_{\mathrm{P} 0}^{2} \frac{\partial \tau_{0}}{\partial z} \frac{\partial \tau_{i j}}{\partial z}=f_{i j}\left(\tau_{0}, \tau_{1}, \tau_{2}, \tau_{3}\right), \\
i, j=1,2,3, \text { and } i \leq j
\end{gathered}
$$

Equation 16 denotes the acoustic eikonal equation for attenuating, elliptically isotropic media. The expressions for the functions in the right sides of equations 17 and 18 are shown in Appendix B. The zeroth-, first-, and second-order traveltime coefficients can be successively obtained from equations 16 to 18 . The analytic solutions to equations 16-18 are shown in Appendix C.

Once we obtain the zeroth-, first-, and second-order traveltime coefficients, we may calculate the complex-valued traveltime from equation 15. Applying Shanks transform (Bender and Orszag [1978], pp. 369-375) to equation 15, the approximation of traveltime is further improved

$$
\tau=T_{0}+\frac{T_{1}^{2}}{T_{1}-T_{2}}
$$

with

$$
T_{0}=\tau_{0}, \quad T_{1}=\sum_{i=1}^{3} \tau_{i} \ell_{i}, \quad T_{2}=\sum_{i, j=1 ; i \leq j}^{3} \tau_{i j} \ell_{i} \ell_{j} .
$$


Setting attenuation anisotropy parameters $\varepsilon_{Q}=\delta_{Q}=0$, equation 19 will reduce to the approximate traveltime for an attenuating VTI medium with the isotropic attenuation coefficient. Setting all perturbation parameters to zero $\left(\eta=\varepsilon_{Q}=\delta_{Q}=0\right)$, equation 19 will reduce to the exact traveltime formula for an attenuating isotropic medium. Setting $A_{\mathrm{P} 0}=0$, the traveltime from equation 19 will become real valued, which corresponds to a nonattenuating VTI medium. In this case, the analytic eikonal solution discussed here will be exactly the same as the one presented in Alkhalifah (2011).

\section{COMPLEX-VALUED TRAVELTIME FOR A HORIZONTAL, ATTENUATING VTI LAYER}

Let us now consider a horizontal, attenuating VTI layer. We denote the source-receiver offset by $x$, and the real and imaginary parts of the reflection traveltime by $t_{R}$ and $t_{I}$. We denote the zero-offset two-way traveltime for the nonattenuating reference of the attenuating VTI layer by $t_{0}=2 z / v_{\mathrm{P} 0}$, where $z$ is the thickness of the layer. From the eikonal solution 19, we may obtain the two-way traveltime as a function of the source-receiver offset. We also consider that $A_{\mathrm{P} 0}$ is very small in practice, which allows us to consider only the first-order influence of $A_{\mathrm{P} 0}$ on the traveltime. In this way, we obtain the expansion for the complex-valued traveltime with respect to the source-receiver offset. Furthermore, we separate the real and imaginary parts of the expansion, which leads to the expansions of the real and imaginary parts of the complex-valued traveltime. Finally, we derive the fourth-order expansions of the squared real and imaginary parts of the two-way reflection traveltime at the zero offset $x=0$,

$$
\begin{gathered}
t_{R}^{2} \approx t_{0}^{2}+\frac{x^{2}}{v_{n}^{2}}-\frac{2 \eta x^{4}}{t_{0}^{2} v_{n}^{4}}, \\
t_{I}^{2} \approx A_{\mathrm{P} 0}^{2}\left(t_{0}^{2}+\frac{x^{2}}{v_{Q}^{2}}-\frac{2 \eta_{Q} x^{4}}{t_{0}^{2} v_{Q}^{4}}\right),
\end{gathered}
$$

with

$$
\begin{gathered}
v_{Q}=\frac{v_{\mathrm{P} 0}(1+2 \delta)}{\sqrt{1+2 \delta+2 \delta_{Q}}}, \\
\eta_{Q}=-\frac{\delta_{Q}^{2}-2(1+2 \delta) \delta_{Q}(1+6 \eta)+2(1+2 \delta)^{2}\left(\varepsilon_{Q}-\eta+2 \varepsilon_{Q} \eta\right)}{2\left(1+2 \delta+2 \delta_{Q}\right)^{2}} .
\end{gathered}
$$

Equation 21 is the same as for acoustic nonattenuating VTI media. Equation 22 involves two new parameters, $v_{Q}$ and $\eta_{Q}$, which have a similar meaning to the NMO velocity $v_{n}$ and the anellipticity parameter $\eta$ for the real part of the complex-valued traveltime. For an acoustic VTI medium with isotropic attenuation coefficient $\left(\varepsilon_{Q}=\delta_{Q}=0\right), v_{Q}$ and $\eta_{Q}$ reduce to $v_{n}$ and $\eta$.

Combining equation 21 and the first-order approximation of the horizontal ray velocity with respect to $A_{\mathrm{P} 0}$, we derive the rational approximation for the real part of the complex-valued traveltime,

$$
t_{R}^{2} \approx t_{0}^{2}+\frac{x^{2}}{v_{n}^{2}}-\frac{2 \eta x^{4}}{t_{0}^{2} v_{n}^{4}\left(1+\xi x^{2}\right)},
$$

with

$$
\xi=\frac{2 \eta}{t_{0}^{2} v_{n}^{4}\left(\frac{1}{v_{n}^{2}}-\frac{1}{v_{h}^{2}}\right)},
$$

where $v_{h}=v_{n} \sqrt{1+2 \eta}$ denotes the velocity of the horizontally propagating quasi P-wave in a nonattenuating VTI medium.

The rational approximation given in equation 25 with equation 26 is the same as the existing rational approximation (Alkhalifah and Tsvankin, 1995; Alkhalifah, 1997) for an acoustic nonattenuating VTI layer. Equation 21 and equation 25 with equation 26 indicate that the real part of the complex-valued traveltime is independent of the attenuation coefficient and the attenuation-anisotropy parameters in Zhu and Tsvankin's (2006) notation under the weak attenuation assumption $\left(A_{\mathrm{P} 0} \ll 1\right)$ and may be described by the existing traveltime approximations for acoustic nonattenuating VTI media.

By analogy with the rational approximation 25 , we derive the rational approximation for the imaginary part of the complex-valued traveltime,

$$
t_{I}^{2} \approx A_{\mathrm{P} 0}^{2}\left(t_{0}^{2}+\frac{x^{2}}{v_{Q}^{2}}-\frac{2 \eta_{Q} x^{4}}{t_{0}^{2} v_{Q}^{4}\left(1+\xi_{Q} x^{2}\right)}\right),
$$

with

$$
\xi_{Q}=\frac{2 \eta_{Q}}{t_{0}^{2} v_{Q}^{4}\left(\frac{1}{v_{Q}^{2}}-\frac{1}{v_{h Q}^{2}}\right)},
$$

where $v_{h Q}$ denotes the inverse of the slope of the curve $t_{I} / A_{\mathrm{P} 0}$ versus source-receiver offset $x$, as $x \rightarrow \infty$; the limit case $x \rightarrow \infty$ corresponds to the $\mathrm{P}$-wave horizontal propagation.

From the acoustic eikonal equation 7, we derive the linearization of $v_{h Q}$ with respect to $A_{\mathrm{P} 0}$,

$$
v_{h Q}=\frac{v_{n} \sqrt{1+2 \eta}}{1+\varepsilon_{Q}} .
$$

If $\varepsilon_{Q}$ becomes zero, then $v_{h Q}$ will be identical to the velocity of the horizontally propagating quasi P-wave in a nonattenuating VTI medium.

Equation 27 indicates that the imaginary part of the complex-valued traveltime, also as a function of source-receiver offset, has a nonhyperbolic shape similar to the traveltime for the acoustic, nonattenuating VTI layer, and it is approximately characterized by five parameters $A_{\mathrm{P} 0}, t_{0}, v_{Q}, \eta_{Q}$, and $\varepsilon_{Q}$, where $A_{\mathrm{P} 0}$ plays the role of a scale factor, which means that the imaginary part of the complexvalued traveltime normalized by $A_{\mathrm{P} 0}$ is characterized by the remaining four parameters; $t_{0}$ describes the zero-offset two-way traveltime in the nonattenuating reference (corresponding to the real parts of complex-valued stiffness coefficients of an attenuating VTI medium); $v_{Q}$ and $\eta_{Q}$ play the roles of the attenuation NMO velocity and the attenuation anellipticity parameter, similar to the roles of the NMO 
velocity $v_{n}$ and the anellipticity parameters $\eta$ for acoustic nonattenuating VTI media. The function $\varepsilon_{Q}$ as an attenuation-anisotropy parameter in the Thomsen-type notation, controls the large-offset behavior of the imaginary part of the complex-valued traveltime normalized by $A_{\mathrm{P} 0}$. For the VTI model with isotropic attenuation coefficient $\left(\varepsilon_{Q}=\delta_{Q}=0\right)$, equation 27 reduces to

$$
t_{I} \approx A_{\mathrm{P} 0} t_{R}
$$

where $t_{R}$ is obtained from equation 25 .

\section{NUMERICAL EXAMPLES}

First, we investigate the influence of the S-wave parameters $v_{\mathrm{S} 0}$ and $A_{\mathrm{S} 0}$ on the P-wave ray velocity and ray attenuation because the complex-valued traveltime is determined by the ray velocity and the ray attenuation for an attenuating anisotropic medium. The ray velocity $V_{\text {ray }}$ and the ray attenuation $A_{\text {ray }}$ are defined by (Vavryčuk, 2007)

$$
\begin{gathered}
V_{\text {ray }}=\frac{v_{R}^{2}+v_{I}^{2}}{v_{R}}, \\
A_{\text {ray }}=-\frac{v_{I}}{v_{R}^{2}+v_{I}^{2}} .
\end{gathered}
$$
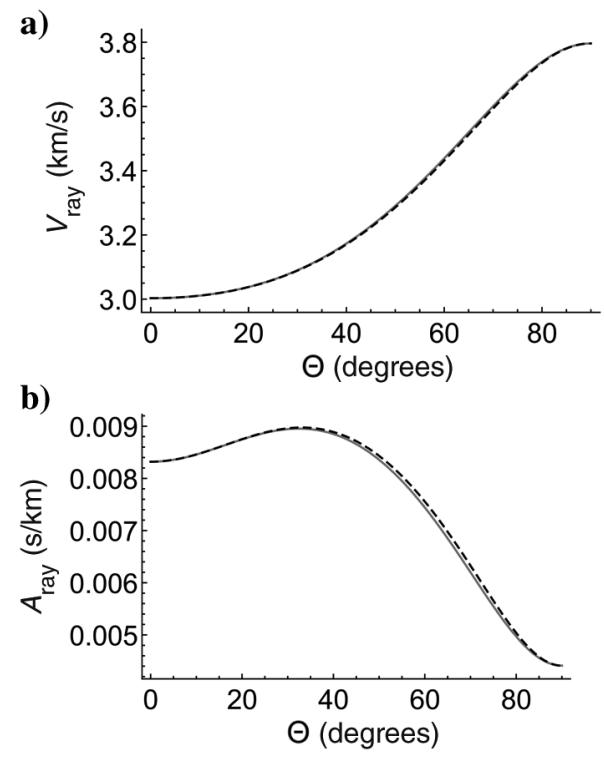

Figure 1. (a) Ray velocity $V_{\text {ray }}$ and (b) ray attenuation $A_{\text {ray }}$ as functions of the ray angle $\Theta$, where the ray angle is measured from the vertical axis to the direction of the homogeneous ray-velocity vector. The gray solid lines correspond to the attenuating VTI model with the parameters $v_{\mathrm{P} 0}=3 \mathrm{~km} / \mathrm{s}, v_{\mathrm{S} 0}=1.5 \mathrm{~km} / \mathrm{s}, \varepsilon=0.3, \delta=0.1, A_{\mathrm{P} 0}=$ 0.02498 (corresponding to $Q_{33}=20$, where $Q_{33}$ denotes the quality factor of P-waves propagating along the vertical axis), $A_{\mathrm{S} 0}=$ 0.03330 (corresponding to $Q_{55}=15$, where $Q_{55}$ denotes the quality factor of $S$-waves propagating along the vertical axis), $\varepsilon_{Q}=-0.33$, and $\delta_{Q}=0.98$. The black dashed lines correspond to the attenuating VTI model with the same parameters except for $v_{\mathrm{S} 0}=0$ and $A_{\mathrm{S} 0}=0$. The values of attenuation parameters $A_{\mathrm{P} 0}, A_{\mathrm{S} 0}, \varepsilon_{Q}$, and $\delta_{Q}$ are from Zhu and Tsvankin (2006).
Here, $v_{R}$ and $v_{I}$ denote the magnitudes of the real and imaginary parts of the complex-valued energy-velocity vector (Vavryčuk, 2007, 2008), and the complex-valued energy-velocity vector is homogeneous, which means that the direction of the vector constructed by the real part of the complex vector coincides with the direction of the vector constructed by the imaginary part of the complex vector. The method of calculating the exact complex-valued energy velocity from a given ray direction for a general attenuating anisotropic medium is summarized in Vavryčuk (2007). In this method, it is essential to solve a system of nonlinear polynomial equations in the unknown vector of phase slowness (Vavryčuk, 2006) for a given ray direction. The complex-valued energy velocity is then calculated from an exact and analytic formula in terms of the vector of phase slowness.

Figure 1 shows that the influences of S-wave parameters $v_{\mathrm{S} 0}$ and $A_{\mathrm{S} 0}$ on the P-wave ray velocity and ray attenuation are very small for VTI media with strong attenuation and strong attenuation anisotropy. This implies that ignoring the parameters $v_{\mathrm{S} 0}$ and $A_{\mathrm{S} 0}$ in the eikonal equation for attenuating VTI media almost does not affect the P-wave complex-valued traveltime.

Second, we test the accuracy of the proposed approximations 15 and 19 for a homogeneous, acoustic attenuating VTI model. In this case, the exact complex-valued traveltime equals the propagation distance divided by the homogenous complex energy velocity (Vavryčuk, 2007). As explained in the first example, the complex-valued energy velocity is exactly calculated by the method discussed in Vavryčuk (2007) for the ray direction formed by the source and a spatial position in a homogeneous model. Figure 2 shows the real and imaginary parts of the exact complex-valued traveltime. The attenuation anisotropy (corresponding to the anisotropy of the imaginary part of the complex-valued traveltime) is far stronger than the wavefront anisotropy (corresponding to the anisotropy of the real part of the complex-valued traveltime). Figures 3 and 4 show the absolute errors in the real and imaginary parts of complex-valued traveltime from approximations 15 and 19. The comparison between Figures 3 and 4 indicates that Shanks transform significantly improves the accuracy of the approximation for the complex-valued traveltime.

Third, we investigate the reflection traveltime for a horizontal, homogeneous, attenuating VTI layer. Figure 5 compares the real parts of the complex-valued traveltimes from the exact solution and the rational approximation 25. The exact solution is calculated by the same method used in the first and second examples. The rational approximation matches the exact solution for small to large offset-depth ratios, which implies that we may still be able to use existing methods such as velocity analysis (Alkhalifah, 1997) to stably invert for the NMO velocity $v_{n}$ and the anellipticity parameter $\eta$ from the P-wave surface data in attenuating VTI models. Figure 6 compares the imaginary part of the complex-valued traveltime from the exact method and the approximations. The secondorder Taylor expansion of the squared imaginary part of the complex-valued traveltime (corresponding to the first two terms in the right side of equation 22) is valid for small offset-depth ratios $(0 \leq x / z \leq 0.8)$. The fourth-order Taylor expansion 22 slightly extends the valid maximum offset-depth ratio up to 1.1 , but the accuracy of this expansion rapidly goes down afterward. The rational approximation 27 is very accurate for offset-depth ratios of less than 1.7 , and the curve of the imaginary part of the complex-valued traveltime from this approximation has the same tendency with the increase in offset-depth ratio as has the curve of the imaginary part of 
the exact complex-valued traveltime. However, the rational approximation 27 becomes inaccurate when the offset-depth ratio is larger than three. Figure 7 shows the influence of attenuation-anisotropy parameters $\varepsilon_{Q}$ and $\delta_{Q}$ on the imaginary part of the reflection traveltime from attenuating VTI layers, in which the reflection traveltime is calculated using the rational approximation 27 . The attenuation-anisotropy parameters $\varepsilon_{Q}$ and $\delta_{Q}$ significantly affect the imaginary part of reflection traveltime for a wide range of offsetdepth ratios $(0.4 \leq x / z \leq 2.8)$.

\section{DISCUSSIONS}

We considered the acoustic approximation (Alkhalifah, 1998, 2000) to derive an acoustic eikonal equation for attenuating VTI media. Although the symmetry axis of the transversely isotropic medium was limited to the vertical, the acoustic eikonal equation for attenuating, transversely isotropic media with a tilted symmetry axis may be obtained by a simple slowness-surface rotation. The acoustic eikonal equation may also be extended to the case of attenuating orthorhombic media. In this case, we will need the Thomsen-type notations (Tsvankin, 1997; Zhu and Tsvankin, 2007) and the acoustic approximation (Alkhalifah, 2003).

The acoustic eikonal equation 7 is not limited to the constant attenuation models. The stiffness coefficients in equation 1 are allowed
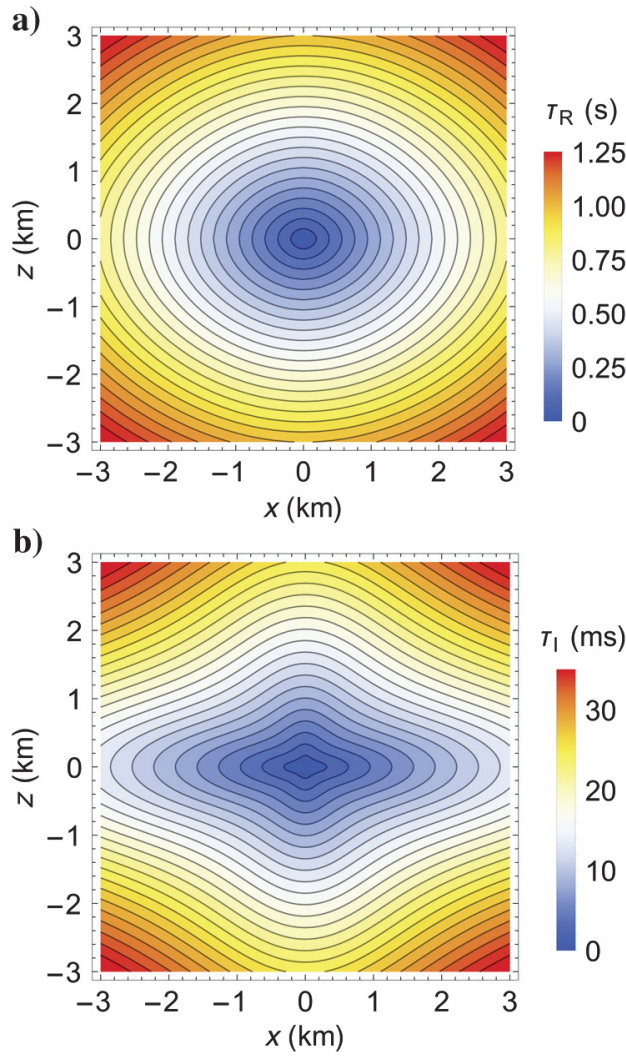

Figure 2. (a) Real and (b) imaginary parts of the exact complex-valued traveltime for a homogeneous, attenuating VTI model. The model parameters are $v_{\mathrm{P} 0}=3 \mathrm{~km} / \mathrm{s}, v_{n}=3.286 \mathrm{~km} / \mathrm{s}, \eta=0.167$, $A_{P 0}=0.02498$ (corresponding to $Q_{33}=20$, where $Q_{33}$ denotes the quality factor of $\mathrm{P}$-waves propagating along the vertical axis), $\varepsilon_{Q}=-0.33$, and $\delta_{Q}=0.98$. The values of attenuation parameters $A_{\mathrm{P} 0}, \varepsilon_{Q}$, and $\delta_{Q}$ are from Zhu and Tsvankin (2006). to be frequency dependent. As illustrated in Zhu and Tsvankin (2006), the parameters in Thomsen (1986) and Zhu and Tsvankin (2006) become frequency dependent if we assume a frequency-dependent attenuating VTI model. In this case, solving the acoustic eikonal equation 7 will provide the traveltime as a function of frequency, the real and imaginary parts of which correspond to the dispersion and frequency-dependent attenuation due to energy absorption.

We have used the perturbation parameters defined in the vector $\ell=\left(\eta, \varepsilon_{Q}, \delta_{Q}\right)^{T}$ to describe the perturbation solution (equation 15) to an attenuating acoustic eikonal equation for a homogeneous attenuating VTI medium. In the perturbation method, the reference medium is taken as an attenuating, elliptically isotropic medium. As explained in the "Introduction" section, however, the numerical method of directly solving the complex eikonal equation, using, for example, finite difference methods, has not been developed even for realistic models with isotropic attenuation. The existing methods designed for nonattenuating media, such as the finite-difference (Vidale, 1988, 1990; van Trier and Symes, 1991), fast-sweeping (Zhang et al., 2006; Luo and Qian, 2012), and fast-marching (Sethian, 1996; Sethian and Vladimirsky, 2001; Alkhalifah, 2011) methods, need to search the minimum traveltime in a heap, to guarantee that the traveltime is always updated along the direction of wave front expansion. The traveltime from the complex eikonal equation is generally complex valued. It is impossible to find the minimum of complex-valued traveltimes. This means that numerical methods developed for nonattenuating media cannot be simply modified to the case of attenuating media. In future work, we will
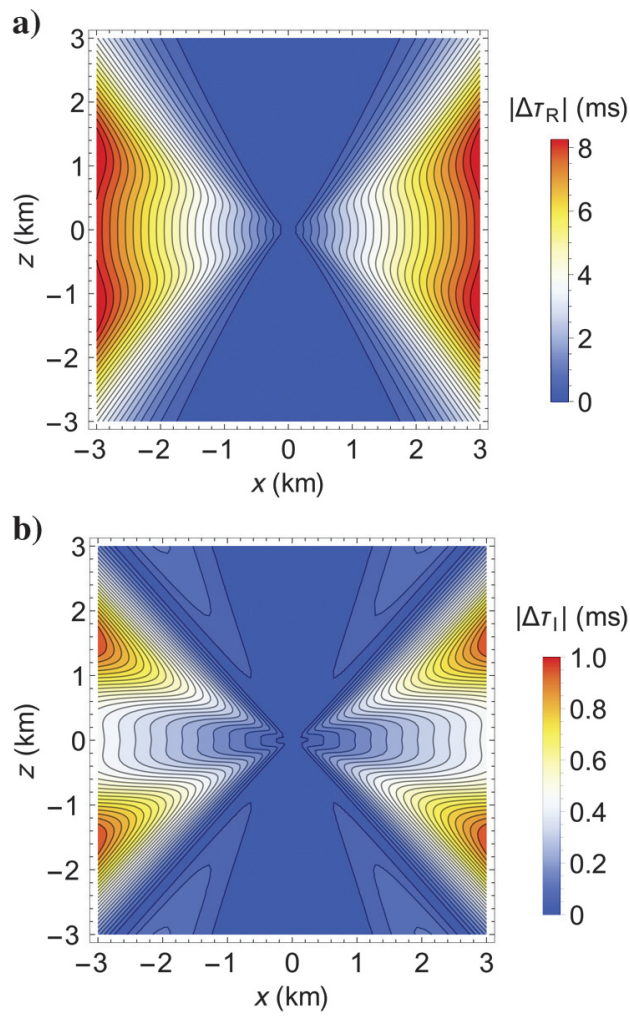

Figure 3. Absolute errors in the (a) real and (b) imaginary parts of the complex-valued traveltime from approximation 15 for a homogeneous, attenuating VTI medium. The model parameters are the same as in Figure 2. 
study further the possibility of direct numerical solutions for the acoustic eikonal equation 7 for realistic attenuating VTI models.

As an alternative, we may combine a perturbation method and Shanks transform to numerically solve the eikonal equation 7 for realistic models under the assumption of weak attenuation. We assume that the attenuation coefficient $A_{\mathrm{P} 0}$ is small enough, and we use it as one of the perturbation parameters. The other perturbation parameters are the same as the parameters that have been used in this paper. Therefore, the new vector $\ell$ composed of perturbation parameters is given by $\ell=\left(A_{\mathrm{P} 0}, \eta, \varepsilon_{Q}, \delta_{Q}\right)^{T}$. As a result, the per-
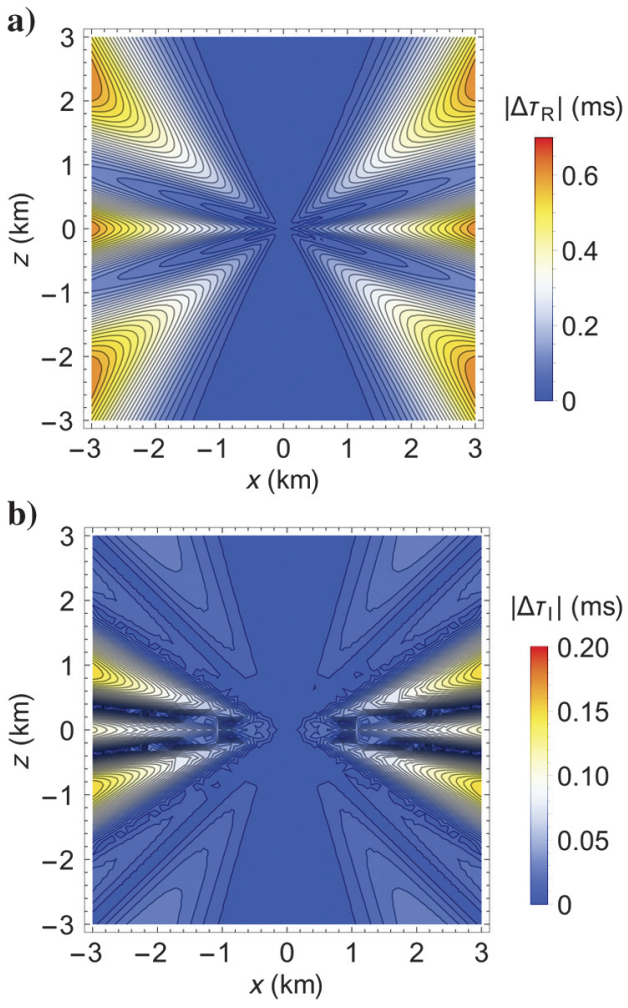

Figure 4. Absolute errors in the (a) real and (b) imaginary parts of the complex-valued traveltime from approximation 19 for a homogeneous, attenuating VTI medium. The model parameters are the same as in Figure 2.

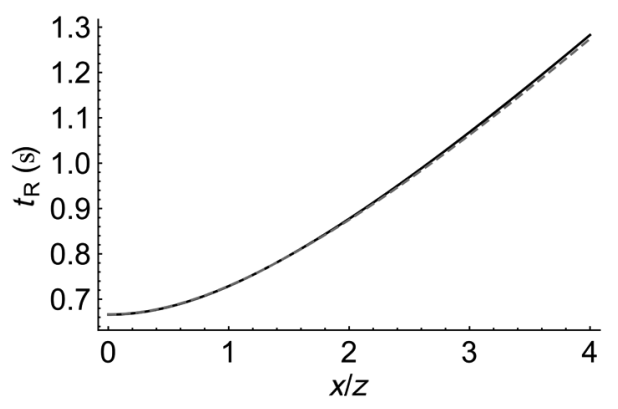

Figure 5. Real part of the complex-valued traveltime as a function of the ratio between the source-receiver offset and the depth of the reflector. The black solid line corresponds to the exact solution; the gray dashed line corresponds to the rational approximation 25 . The layer thickness is $z=1 \mathrm{~km}$. The medium parameters are the same as in Figure 2. turbation expansion of the complex-valued traveltime with respect to the new perturbation parameters may be described by using the same method introduced in this paper. The zeroth-, first-, and second-order traveltime coefficients are then controlled by the partial differential equations similar to equations 16-18. In this case, the zeroth-order traveltime coefficient corresponds to the eikonal equation for nonattenuating, elliptically isotropic media, which can be solved by the fast-marching method (Alkhalifah, 2011), for example. The first- and second-order traveltime coefficients are calculated by solving the corresponding first-order partial differential equations in the order of updating traveltimes in the discretized reference medium. Once all the traveltime coefficients are obtained, we may use the Shanks transform to calculate the complex-valued traveltime. The nonattenuating version of this algorithm is presented in Alkhalifah (2011).

We have derived a nonhyperbolic approximation (equation 27) for the imaginary part of the complex-valued reflection traveltime.

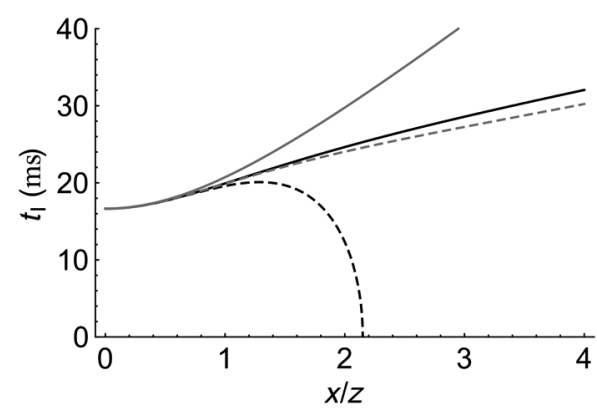

Figure 6. Imaginary part of the complex-valued traveltime as a function of the ratio between the source-receiver offset and the depth of the reflector. The black solid line corresponds to the exact solution; the gray solid line corresponds to the second-order approximation described by the first two terms in the right side of equation 22; the black dashed line corresponds to the fourth-order approximation 22 ; and the gray dashed line corresponds to the rational approximation 27. The model parameters are the same as in Figure 5.

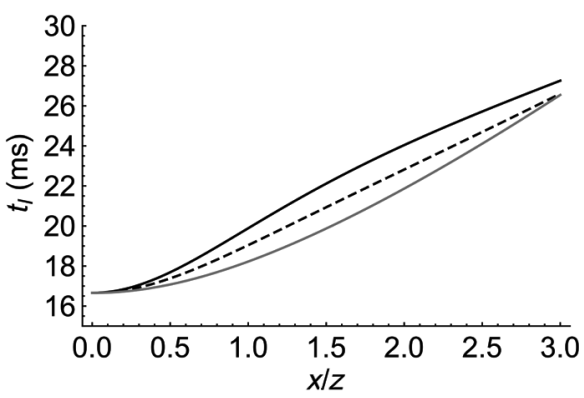

Figure 7. Influence of attenuation-anisotropy parameters on the imaginary part of the complex-valued traveltime from three single-layer VTI models. The three models share the layer thickness $z=$ $1 \mathrm{~km}$ and the medium parameters $v_{\mathrm{P} 0}=3 \mathrm{~km} / \mathrm{s}, v_{n}=3.286 \mathrm{~km} / \mathrm{s}$, $\eta=0.167, A_{\mathrm{P} 0}=0.02498$ (corresponding to $Q_{33}=20$, where $Q_{33}$ denotes the quality factor of $\mathrm{P}$-waves propagating along the vertical axis). The black solid line corresponds to the VTI model with strong attenuation anisotropy $\left(\varepsilon_{Q}=-0.33\right.$ and $\left.\delta_{Q}=0.98\right)$. The black dashed line corresponds to the VTI model with relatively weak attenuation anisotropy $\left(\varepsilon_{Q}=-0.165\right.$ and $\left.\delta_{Q}=0.49\right)$. The gray solid line corresponds to the VTI model with isotropic attenuation coefficient $\left(\varepsilon_{Q}=0\right.$ and $\left.\delta_{Q}=0\right)$. The imaginary part of the complex-valued traveltime is calculated using the rational approximation 27 . 
Following Behura and Tsvankin (2009), this approximation can be used in conjunction with layer stripping to invert for the parameters $A_{\mathrm{P} 0}, v_{Q}, \eta_{Q}$, and $\varepsilon_{Q}$ for a single horizontal layer. From the third example in the "Numerical examples" section, we may expect that (1) the parameters $A_{\mathrm{P} 0}$ and $v_{Q}$ are estimated from small-offset data (corresponding to the ratio of the source-receiver offset to the layer thickness less than 0.8 ); (2) the parameter $\eta_{Q}$ is estimated from slightly larger offset data (corresponding to the ratio of the sourcereceiver offset to the layer thickness approximately 1.0); (3) estimating the parameter $\varepsilon_{Q}$ needs moderate-offset data (corresponding to the ratio of the source-receiver offset to the layer thickness between 1.0 and 2.0); and (4) the parameter $\varepsilon_{Q}$ may also be coarsely estimated from the slope of the imaginary part of the complex-valued traveltime at large offsets (corresponding to the ratio of the source-receiver offset to the layer thickness larger than 3.0).

\section{CONCLUSION}

The acoustic eikonal equation is derived based on the assumption that the influence of the S-wave velocity parameter $v_{\mathrm{S} 0}$ in Thomsen's notation and the S-wave attenuation coefficient $A_{\mathrm{S} 0}$ in Zhu and Tsvankin's notation on the complex-valued traveltime of quasi P-waves in attenuating VTI media is negligible. Combining perturbation theory and Shanks transform leads to an accurate analytic solution to the acoustic eikonal equation for homogeneous attenuating VTI media. For a horizontal homogeneous VTI layer with strong attenuation, the influence of the $\mathrm{P}$-wave attenuation coefficient $A_{\mathrm{P} 0}$ in Zhu and Tsvankin's notation on the real part of the complex-valued traveltime is weak to the point that we may use the existing nonhyperbolic approximations such as the rational approximation to describe the real part of the complex-valued traveltime; the imaginary part of the complex-valued traveltime, as a function of sourcereceiver offset, also has a nonhyperbolic shape, which is expressed by the rational approximation.

\section{ACKNOWLEDGMENTS}

Q. Hao thanks the Rock and Seismic (ROSE) project for support and A. Stovas for helpful discussion on attenuation anisotropy. T. Alkhalifah thanks KAUST for its support. We also thank the assistant editor J. Etgen, associate editor Y. Liu, and three anonymous reviewers for many valuable suggestions.

\section{APPENDIX A}

\section{PARAMETERIZATION FOR ATTENUATING VTI MEDIA}

Here, we show the parameterization for an attenuating VTI medium. As illustrated in equation 1, the stiffness coefficients of an attenuating VTI medium are denoted by $c_{i j}=c_{i j}^{R}-i c_{i j}^{I}$. The $\mathbf{Q}$ matrix is defined as $Q_{i j}=c_{i j}^{R} / c_{i j}^{I}$, where the summation over $i$ and $j$ does not exist. Note that the definition for the $\mathbf{Q}$ matrix corresponds to the stiffness coefficients $c_{i j}=c_{i j}^{R}-i c_{i j}^{I}$ and traveltime $\tau=\tau_{R}+i \tau_{I}$, which is slightly different from that of Zhu and Tsvankin (2006). Besides, the density of the medium is denoted by $\rho$. We combine Thomsen's (1986) notation and Zhu and Tsvankin's (2006) notation to parameterize an attenuating VTI medium. Thomsen's (1986) notation is used to describe the wave velocities in the nonattenuating reference of the attenuating VTI medium, in which the nonattenuating reference corresponds to the real part of the stiffness coefficients of the attenuating VTI medium. Zhu and Tsvankin's (2006) notation is used to describe attenuation coefficients of homogeneous planes waves in an attenuating VTI medium. Combining Thomsen's (1986) notation and Zhu and Tsvankin's (2006) notation, an attenuating VTI medium is parameterized by the following parameters:

1) $v_{\mathrm{P} 0}$ : the velocity of the vertically propagating $\mathrm{P}$-wave in the nonattenuating VTI reference with stiffness coefficients $c_{i j}^{R}$ :

$$
v_{\mathrm{P} 0} \equiv \sqrt{\frac{c_{33}^{R}}{\rho}}
$$

2) $v_{\mathrm{S} 0}$ : the velocity of the vertically propagating $\mathrm{S}$-wave in the nonattenuating VTI reference with stiffness coefficients $c_{i j}^{R}$ :

$$
v_{\mathrm{S} 0} \equiv \sqrt{\frac{c_{55}^{R}}{\rho}}
$$

3) $\varepsilon, \delta, \gamma$ : the Thomsen (1986) parameters for the nonattenuating VTI reference with stiffness coefficients $c_{i j}^{R}$ :

$$
\begin{gathered}
\varepsilon \equiv \frac{c_{11}^{R}-c_{33}^{R}}{2 c_{33}^{R}}, \\
\delta \equiv \frac{\left(c_{13}^{R}+c_{55}^{R}\right)^{2}-\left(c_{33}^{R}-c_{55}^{R}\right)^{2}}{2 c_{33}^{R}\left(c_{33}^{R}-c_{55}^{R}\right)},
\end{gathered}
$$

4) $A_{\mathrm{P} 0}$ : the P-wave attenuation coefficient in the symmetry (vertical) direction:

$$
A_{\mathrm{P} 0} \equiv Q_{33}\left(\sqrt{1+\frac{1}{Q_{33}^{2}}}-1\right)
$$

5) $A_{\mathrm{S} 0}$ : the $\mathrm{S}$-wave attenuation coefficient in the symmetry (vertical) direction:

$$
A_{\mathrm{S} 0} \equiv Q_{55}\left(\sqrt{1+\frac{1}{Q_{55}^{2}}}-1\right)
$$

6) $\varepsilon_{Q}, \delta_{Q}, \gamma_{Q}$ : the Thomsen parameters for attenuation anisotropy:

$$
\varepsilon_{Q} \equiv \frac{\frac{1}{Q_{11}}-\frac{1}{Q_{33}}}{\frac{1}{Q_{33}}}=\frac{Q_{33}-Q_{11}}{Q_{11}},
$$




$$
\begin{gathered}
\delta_{Q} \equiv \frac{\frac{Q_{33}-Q_{55}}{Q_{55}} c_{55}^{R} \frac{\left(c_{13}^{R}+c_{33}^{R}\right)^{2}}{\left(c_{33}^{R}-c_{55}^{R}\right)}+2 \frac{Q_{33}-Q_{13}}{Q_{13}} c_{13}^{R}\left(c_{13}^{R}+c_{55}^{R}\right)}{c_{33}^{R}\left(c_{33}^{R}-c_{55}^{R}\right)}, \\
\gamma_{Q} \equiv \frac{\frac{1}{Q_{66}}-\frac{1}{Q_{55}}}{\frac{1}{Q_{55}}}=\frac{Q_{55}-Q_{66}}{Q_{66}} .
\end{gathered}
$$

Here, equations A-1-A-5 are from Thomsen (1986), and equations A-6-A-10 are from Zhu and Tsvankin (2006); $\varepsilon_{Q}$ denotes the fractional difference between the P-wave attenuation coefficients in the horizontal and vertical directions; $\delta_{Q}$ denotes the second-order derivative of the $\mathrm{P}$-wave attenuation coefficient at the phase angle equal to zero, where the phase angle is measured from the phase propagation direction to the symmetry (vertical) direction; and $\gamma_{Q}$ denotes the fractional difference between the $\mathrm{SH}$-wave attenuation coefficients in the horizontal and vertical directions.

\section{APPENDIX B}

\section{THE RIGHT SIDES OF EQUATIONS 17 AND 18}

We show the expressions for the functions in the right sides of equations 17 and 18 .

The functions in the right sides of equation 17:

$$
\begin{gathered}
f_{1}\left(\tau_{0}\right)=-v_{n}^{2}\left(\frac{\partial \tau_{0}}{\partial x}\right)^{2}+c v_{\mathrm{P} 0}^{2} v_{n}^{2}\left(\frac{\partial \tau_{0}}{\partial x}\right)^{2}\left(\frac{\partial \tau_{0}}{\partial z}\right)^{2}, \\
f_{2}\left(\tau_{0}\right)=a v_{n}^{2}\left(\frac{\partial \tau_{0}}{\partial x}\right)^{2}-b v_{\mathrm{P} 0}^{2} v_{n}^{2}\left(\frac{\partial \tau_{0}}{\partial x}\right)^{2}\left(\frac{\partial \tau_{0}}{\partial z}\right)^{2}, \\
f_{3}\left(\tau_{0}\right)=b v_{\mathrm{P} 0}^{4}\left(\frac{\partial \tau_{0}}{\partial x}\right)^{2}\left(\frac{\partial \tau_{0}}{\partial z}\right)^{2},
\end{gathered}
$$

with

$$
\begin{gathered}
a=\frac{i A_{\mathrm{P} 0}}{\left(1-i A_{\mathrm{P} 0}\right)^{2}}, \\
b=\frac{i A_{\mathrm{P} 0}}{1-A_{\mathrm{P} 0}^{2}}, \\
c=\frac{\left(1-i A_{\mathrm{P} 0}\right)^{2}}{1-A_{\mathrm{P} 0}^{2}} .
\end{gathered}
$$

The functions in right sides of equation 18:

$$
\begin{gathered}
f_{11}\left(\tau_{0}, \tau_{1}, \tau_{2}, \tau_{3}\right)=-2 v_{n}^{2} \frac{\partial \tau_{0}}{\partial x} \frac{\partial \tau_{1}}{\partial x}+2 c v_{\mathrm{P} 0}^{2} v_{n}^{2} \frac{\partial \tau_{0}}{\partial x}\left(\frac{\partial \tau_{0}}{\partial z}\right)^{2} \frac{\partial \tau_{1}}{\partial x} \\
+2 c v_{\mathrm{P} 0}^{2} v_{n}^{2}\left(\frac{\partial \tau_{0}}{\partial x}\right)^{2} \frac{\partial \tau_{0}}{\partial z} \frac{\partial \tau_{1}}{\partial z}-\frac{1}{2} v_{n}^{2}\left(\frac{\partial \tau_{1}}{\partial x}\right)^{2}-\frac{1}{2} v_{\mathrm{P} 0}^{2}\left(\frac{\partial \tau_{1}}{\partial z}\right)^{2}
\end{gathered}
$$

$$
\begin{gathered}
f_{22}\left(\tau_{0}, \tau_{1}, \tau_{2}, \tau_{3}\right)=2 a v_{n}^{2} \frac{\partial \tau_{0}}{\partial x} \frac{\partial \tau_{2}}{\partial x}-2 b v_{\mathrm{P} 0}^{2} v_{n}^{2} \frac{\partial \tau_{0}}{\partial x}\left(\frac{\partial \tau_{0}}{\partial z}\right)^{2} \frac{\partial \tau_{2}}{\partial x} \\
-2 b v_{\mathrm{P} 0}^{2} v_{n}^{2}\left(\frac{\partial \tau_{0}}{\partial x}\right)^{2} \frac{\partial \tau_{0}}{\partial z} \frac{\partial \tau_{2}}{\partial z}-\frac{1}{2} v_{n}^{2}\left(\frac{\partial \tau_{2}}{\partial x}\right)^{2}-\frac{1}{2} v_{\mathrm{P} 0}^{2}\left(\frac{\partial \tau_{2}}{\partial z}\right)^{2}
\end{gathered}
$$

$$
\begin{aligned}
& f_{33}\left(\tau_{0}, \tau_{1}, \tau_{2}, \tau_{3}\right)=-\frac{1}{2} a b \frac{v_{\mathrm{P} 0}^{6}}{v_{n}^{2}}\left(\frac{\partial \tau_{0}}{\partial x}\right)^{2}\left(\frac{\partial \tau_{0}}{\partial z}\right)^{2} \\
& +2 b v_{\mathrm{P} 0}^{4} \frac{\partial \tau_{0}}{\partial x}\left(\frac{\partial \tau_{0}}{\partial z}\right)^{2} \frac{\partial \tau_{3}}{\partial x} \\
& +2 b v_{\mathrm{P} 0}^{4}\left(\frac{\partial \tau_{0}}{\partial x}\right)^{2} \frac{\partial \tau_{0}}{\partial z} \frac{\partial \tau_{3}}{\partial z}-\frac{1}{2} v_{n}^{2}\left(\frac{\partial \tau_{3}}{\partial x}\right)^{2}-\frac{1}{2} v_{\mathrm{P} 0}^{2}\left(\frac{\partial \tau_{3}}{\partial z}\right)^{2}
\end{aligned}
$$

$$
\begin{aligned}
& f_{12}\left(\tau_{0}, \tau_{1}, \tau_{2}, \tau_{3}\right)=2 a v_{n}^{2}\left(\frac{\partial \tau_{0}}{\partial x}\right)^{2}+2 a v_{n}^{2} \frac{\partial \tau_{0}}{\partial x} \frac{\partial \tau_{1}}{\partial x}-2 v_{n}^{2} \frac{\partial \tau_{0}}{\partial x} \frac{\partial \tau_{2}}{\partial x} \\
& -2 b v_{\mathrm{P} 0}^{2} v_{n}^{2}\left(\frac{\partial \tau_{0}}{\partial x}\right)^{2}\left(\frac{\partial \tau_{0}}{\partial z}\right)^{2}-2 b v_{\mathrm{P} 0}^{2} v_{n}^{2} \frac{\partial \tau_{0}}{\partial x}\left(\frac{\partial \tau_{0}}{\partial z}\right)^{2} \frac{\partial \tau_{1}}{\partial x} \\
& -2 b v_{\mathrm{P} 0}^{2} v_{n}^{2}\left(\frac{\partial \tau_{0}}{\partial x}\right)^{2} \frac{\partial \tau_{0}}{\partial z} \frac{\partial \tau_{1}}{\partial z} \\
& +2 c v_{\mathrm{P} 0}^{2} v_{n}^{2} \frac{\partial \tau_{0}}{\partial x}\left(\frac{\partial \tau_{0}}{\partial z}\right)^{2} \frac{\partial \tau_{2}}{\partial x}+2 c v_{\mathrm{P} 0}^{2} v_{n}^{2}\left(\frac{\partial \tau_{0}}{\partial x}\right)^{2} \frac{\partial \tau_{0}}{\partial z} \frac{\partial \tau_{2}}{\partial z} \\
& -v_{n}^{2} \frac{\partial \tau_{1}}{\partial x} \frac{\partial \tau_{2}}{\partial x}-v_{\mathrm{P} 0}^{2} \frac{\partial \tau_{1}}{\partial z} \frac{\partial \tau_{2}}{\partial z},
\end{aligned}
$$

$$
\begin{aligned}
& f_{13}\left(\tau_{0}, \tau_{1}, \tau_{2}, \tau_{3}\right)=2 b v_{\mathrm{P} 0}^{4} \frac{\partial \tau_{0}}{\partial x}\left(\frac{\partial \tau_{0}}{\partial z}\right)^{2} \frac{\partial \tau_{1}}{\partial x} \\
& +2 b v_{\mathrm{P} 0}^{4}\left(\frac{\partial \tau_{0}}{\partial x}\right)^{2} \frac{\partial \tau_{0}}{\partial z} \frac{\partial \tau_{1}}{\partial z}-2 v_{n}^{2} \frac{\partial \tau_{0}}{\partial x} \frac{\partial \tau_{3}}{\partial x} \\
& +2 c v_{\mathrm{P} 0}^{2} v_{n}^{2} \frac{\partial \tau_{0}}{\partial x}\left(\frac{\partial \tau_{0}}{\partial z}\right)^{2} \frac{\partial \tau_{3}}{\partial x}+2 c v_{\mathrm{P} 0}^{2} v_{n}^{2}\left(\frac{\partial \tau_{0}}{\partial x}\right)^{2} \frac{\partial \tau_{0}}{\partial z} \frac{\partial \tau_{3}}{\partial z} \\
& -v_{n}^{2} \frac{\partial \tau_{1}}{\partial x} \frac{\partial \tau_{3}}{\partial x}-v_{\mathrm{P} 0}^{2} \frac{\partial \tau_{1}}{\partial z} \frac{\partial \tau_{3}}{\partial z}
\end{aligned}
$$




$$
\begin{gathered}
f_{23}\left(\tau_{0}, \tau_{1}, \tau_{2}, \tau_{3}\right)=2 a v_{n}^{2} \frac{\partial \tau_{0}}{\partial x} \frac{\partial \tau_{3}}{\partial x}+2 b v_{\mathrm{P} 0}^{4} \frac{\partial \tau_{0}}{\partial x}\left(\frac{\partial \tau_{0}}{\partial z}\right)^{2} \frac{\partial \tau_{2}}{\partial x} \\
+2 b v_{\mathrm{P} 0}^{4}\left(\frac{\partial \tau_{0}}{\partial x}\right)^{2} \frac{\partial \tau_{0}}{\partial z} \frac{\partial \tau_{2}}{\partial z} \\
-2 b v_{\mathrm{P} 0}^{2} v_{n}^{2} \frac{\partial \tau_{0}}{\partial x}\left(\frac{\partial \tau_{0}}{\partial z}\right)^{2} \frac{\partial \tau_{3}}{\partial x}-2 b v_{\mathrm{P} 0}^{2} v_{n}^{2}\left(\frac{\partial \tau_{0}}{\partial x}\right)^{2} \frac{\partial \tau_{0}}{\partial z} \frac{\partial \tau_{3}}{\partial z} \\
-v_{n}^{2} \frac{\partial \tau_{2}}{\partial x} \frac{\partial \tau_{3}}{\partial x}-v_{\mathrm{P} 0}^{2} \frac{\partial \tau_{2}}{\partial z} \frac{\partial \tau_{3}}{\partial z},
\end{gathered}
$$

where $a, b$, and $c$ are given by equations B-4-B-6.

\section{APPENDIX C}

\section{THE TRAVELTIME COEFFICIENTS FOR A HOMOGENEOUS ATTENUATING VTI MEDIUM}

We show the analytic solutions to equations $16-18$ for a homogeneous attenuating VTI medium.

The solution to equation 16 is given by

$$
\tau_{0}=\frac{\sqrt{\left(1-A_{\mathrm{P} 0}^{2}\right)\left(v_{\mathrm{P} 0}^{2} x^{2}+v_{n}^{2} z^{2}\right)}}{\left(1-i A_{\mathrm{P} 0}\right) v_{n} v_{\mathrm{P} 0}} .
$$

The solutions to equation 17 are as follows:

$$
\begin{gathered}
\tau_{1}=-\frac{x^{4} v_{\mathrm{P} 0}^{3} \sqrt{1-A_{\mathrm{P} 0}^{2}}}{v_{n}\left(1-i A_{\mathrm{P} 0}\right)\left(v_{\mathrm{P} 0}^{2} x^{2}+v_{n}^{2} z^{2}\right)^{3 / 2}}, \\
\tau_{2}=\frac{i A_{\mathrm{P} 0} \sqrt{1-A_{\mathrm{P} 0}^{2}} v_{\mathrm{P} 0}^{3} x^{4}}{\left(1-i A_{\mathrm{P} 0}\right)^{3} v_{n}\left(v_{\mathrm{P} 0}^{2} x^{2}+v_{n}^{2} z^{2}\right)^{3 / 2}}, \\
\tau_{3}=\frac{i A_{\mathrm{P} 0} \sqrt{1-A_{\mathrm{P} 0}^{2}} v_{\mathrm{P} 0}^{3} x^{2} z^{2}}{\left(1-i A_{\mathrm{P} 0}\right)^{3} v_{n}\left(v_{\mathrm{P} 0}^{2} x^{2}+v_{n}^{2} z^{2}\right)^{3 / 2}} .
\end{gathered}
$$

The solutions to equation 18 are as follows:

$$
\begin{gathered}
\tau_{11}=\frac{3 \sqrt{1-A_{\mathrm{P} 0}^{2}} v_{\mathrm{P} 0}^{5} x^{6}\left(v_{\mathrm{P} 0}^{2} x^{2}+4 v_{n}^{2} z^{2}\right)}{2\left(1-i A_{\mathrm{P} 0}\right) v_{n}\left(v_{\mathrm{P} 0}^{2} x^{2}+v_{n}^{2} z^{2}\right)^{7 / 2}}, \\
\tau_{22}=-\frac{3 A_{\mathrm{P} 0}^{2} \sqrt{1-A_{\mathrm{P} 0}^{2}} v_{\mathrm{P} 0}^{5} x^{6}\left(v_{\mathrm{P} 0}^{2} x^{2}+4 v_{n}^{2} z^{2}\right)}{2\left(1-i A_{\mathrm{P} 0}\right)^{5} v_{n}\left(v_{\mathrm{P} 0}^{2} x^{2}+v_{n}^{2} z^{2}\right)^{7 / 2}}, \quad(\mathrm{C}-6) \\
\tau_{33}=-\frac{3 A_{\mathrm{P} 0}^{2} \sqrt{1-A_{\mathrm{P} 0}^{2}} v_{\mathrm{P} 0}^{5} x^{2} z^{2}\left(v_{\mathrm{P} 0}^{4} x^{4}-v_{\mathrm{P} 0}^{2} v_{n}^{2} x^{2} z^{2}+v_{n}^{4} z^{4}\right)}{2\left(1-i A_{\mathrm{P} 0}\right)^{5} v_{n}^{3}\left(v_{\mathrm{P} 0}^{2} x^{2}+v_{n}^{2} z^{2}\right)^{7 / 2}},
\end{gathered}
$$

$$
\tau_{12}=-\frac{i A_{\mathrm{P} 0} \sqrt{1-A_{\mathrm{P} 0}^{2}} v_{\mathrm{P} 0}^{3} x^{4}\left(v_{\mathrm{P} 0}^{4} x^{4}+8 v_{\mathrm{P} 0}^{2} v_{n}^{2} x^{2} z^{2}-2 v_{n}^{4} z^{4}\right)}{\left(1-i A_{\mathrm{P} 0}\right)^{3} v_{n}\left(v_{\mathrm{P} 0}^{2} x^{2}+v_{n}^{2} z^{2}\right)^{7 / 2}},
$$

$$
\tau_{13}=\frac{3 i A_{\mathrm{P} 0} \sqrt{1-A_{\mathrm{P} 0}^{2}} v_{\mathrm{P} 0}^{5} x^{4} z^{2}\left(v_{\mathrm{P} 0}^{2} x^{2}-2 v_{n}^{2} z^{2}\right)}{\left(1-i A_{\mathrm{P} 0}\right)^{3} v_{n}\left(v_{\mathrm{P} 0}^{2} x^{2}+v_{n}^{2} z^{2}\right)^{7 / 2}},
$$

$$
\tau_{23}=\frac{3 A_{\mathrm{P} 0}^{2} \sqrt{1-A_{\mathrm{P} 0}^{2}} v_{\mathrm{P} 0}^{5} x^{4} z^{2}\left(v_{\mathrm{P} 0}^{2} x^{2}-2 v_{n}^{2} z^{2}\right)}{\left(1-i A_{\mathrm{P} 0}\right)^{5} v_{n}\left(v_{\mathrm{P} 0}^{2} x^{2}+v_{n}^{2} z^{2}\right)^{7 / 2}} .
$$

\section{REFERENCES}

Aki, K., and P. G. Richards, 2002, Quantitative seismology: (2nd ed.): University Science Books.

Alkhalifah, T., 1997, Velocity analysis using nonhyperbolic moveout in transversely isotropic media: Geophysics, 62, 1839-1854, doi: 10 $1190 / 1.1444285$

Alkhalifah, T., 1998, Acoustic approximations for seismic processing in transversely isotropic media: Geophysics, 63, 623-631, doi: 10.1190/1 .1444361.

Alkhalifah, T., 2000, An acoustic wave equation for anisotropic media: Geophysics, 65, 1239-1250, doi: 10.1190/1.1444815.

Alkhalifah, T., 2003, An acoustic wave equation for orthorhombic anisotropy: Geophysics, 68, 1169-1172, doi: 10.1190/1.1598109.

Alkhalifah, T., 2011, Scanning anisotropy parameters in complex media: Geophysics, 76, no. 2, U13-U22, doi: 10.1190/1.3553015.

Alkhalifah, T., and I. Tsvankin, 1995, Velocity analysis for transversely isotropic media: Geophysics, 60, 1550-1566, doi: 10.1190/1.1443888.

Amodei, D., H. Keers, W. Vasco, and L. Johnson, 2006, Computation of uniform wave forms using complex rays: Physical Review E, 73, 1-14.

Behura, J., and I. Tsvankin, 2009, Estimation of interval anisotropic attenuation from reflection data: Geophysics, 74, no. 6, A69-A74, doi: 10.1190/ 1.3191733 .

Behura, J., I. Tsvankin, E. Jenner, and A. Calvert, 2012, Estimation of interval velocity and attenuation anisotropy from reflection data at Coronation Field: The Leading Edge, 31, 580-587, doi: 10.1190/tle31050580.1.

Bender, C. M., and S. A. Orszag, 1978, Advanced mathematical methods for scientists and engineers: McGraw-Hill.

Ben-Menahem, A., and S. J. Singh, 1981, Seismic waves and sources: Springer.

Best, A. I., J. Sothcott, and C. McCann, 2007, A laboratory study of seismic velocity and attenuation anisotropy in near-surface sedimentary rocks: Geophysical Prospecting, 55, 609-625, doi: 10.1111/j.1365-2478.2007 .00642.x.

Borcherdt, R. D., 2009, Viscoelastic waves in layered media: Cambridge University Press.

Carcione, J. M., 2015, Wave fields in real media: Theory and numerical simulation of wave propagation in anisotropic, anelastic, porous and electromagnetic media: Handbook of Geophysical exploration (3rd ed.): Elsevier

Carter, A. J., and J.-M. Kendall, 2006, Attenuation anisotropy and the relative frequency content of split shear waves: Geophysical Journal International, 165, 865-874, doi: 10.1111/j.1365-246X.2006.02929.x.

Červený, V., 2001, Seismic ray theory: Cambridge University Press.

Červený, V., and I. Pšenčík, 2005, Plane waves in viscoelastic anisotropic media - I. Theory: Geophysical Journal International, 161, 197-212, doi: 10.1111/j.1365-246X.2005.02589.x.

Červený, V., and I. Pšenčík, 2006, Energy flux in viscoelastic anisotropic media: Geophysical Journal International, 166, 1299-1317, doi: 10 $.1111 / \mathrm{j} .1365-246 X .2006 .03057 . x$

Červený, V., and I. Pšenčík, 2009, Perturbation Hamiltonians in heterogeneous anisotropic weakly dissipative media: Geophysical Journal International, 178, 939-949, doi: 10.1111/j.1365-246X.2009.04218.x.

Chapman, S. J., J. M. H. Lawry, J. R. Ockendon, and R. H. Tew, 1999, On the theory of complex rays: SIAM Review, 41, 417-509, doi: 10.1137/ S0036144599352058.

Clark, R. A., P. M. Benson, A. J. Carter, and C. A. G. Moreno, 2009, Anisotropic P-wave attenuation measured from a multi-azimuth surface seismic 
reflection survey: Geophysical Prospecting, 57, 835-845, doi: 10.1111/j .1365-2478.2008.00772.x.

Fedorov, F. I., 1968, Theory of elastic waves in crystals: Springer.

Gajewski, D., and I. Pšenčík, 1992, Vector wavefields for weakly attenuating anisotropic media by the ray method: Geophysics, 57, 27-38, doi: 10 $.1190 / 1.1443186$

Hanyga, A., and M. Seredyňska, 2000, Ray tracing in elastic and viscoelastic media: Pure and Applied Geophysics, 157, 679-717, doi: 10.1007/ PL00001114.

Hearn, D. J., and E. S. Krebes, 1990a, On computing ray-synthetic seismograms for anelastic media using complex rays: Geophysics, 55, 422-432, doi: $10.1190 / 1.1442851$

Hearn, D. J., and E. S. Krebes, 1990b, Complex rays applied to wave propagation in a viscoelastic medium: Pure and Applied Geophysics, 132, 401415, doi: $10.1007 / \mathrm{BF} 00874371$.

Klimeš, M., and L. Klimeš, 2011, Perturbation expansion of complex-valued traveltime along real-valued reference rays: Geophysics Journal International, 186, 751-759, doi: 10.1111/j.1365-246X.2011.05054.x.

Kravtsov, Y. A., G. W. Forbes, and A. A. Asatryan, 1999, Theory and applications of complex rays, in E. Wolf, ed., Progress in optics: Elsevier 39, $1-62$.

Krebes, E. S., and M. A. Slawinski, 1991, On ray tracing in an elastic-anelastic medium: Bulletin of the Seismological Society of America, 81, 667-686.

Le, L. H. T., E. S. Krebes, and G. E. Quiroga-Goode, 1994, Synthetic seismograms for $\mathrm{SH}$ waves in anelastic transversely isotropic media: Geophysical Journal International, 116, 598-604, doi: 10.1111/j.1365-246X .1994.tb03283.x.

Luo, S., and J. Qian, 2012, Fast sweeping methods for factored anisotropic eikonal equations: Multiplicative and additive factors. Journal of Scientific Computing, 52, 360-382, doi: 10.1007/s10915-011-9550-y.

Rasolofosaon, P. N. J., 2010, Generalized anisotropy parameters and approximations of attenuations and velocities in viscoelastic media of arbitrary anisotropy type - Theoretical and experimental aspects: Geophysical Prospecting, 58, 637-655, doi: 10.1111/j.1365-2478.2009.00863.x.

Sethian, J. A., 1996, A fast marching level set method for monotonically advancing fronts: Proceedings of the National Academy of Sciences of the United States of America, 93, 1591-1595, doi: 10.1073/pnas.93.4.1591.

Sethian, J. A., and A. Vladimirsky, 2001, Ordered upwind methods for static Hamilton-Jacobi equations: Proceedings of the National Academy of Sciences, 98, 11069-11074, doi: 10.1073/pnas.201222998.

Shekar, B., and I. Tsvankin, 2012, Anisotropic attenuation analysis of crosshole data generated during hydraulic fracturing: The Leading Edge, 31, 588-593, doi: 10.1190/tle31050588.1.

Stovas, A., and T. Alkhalifah, 2012, A new traveltime approximation for TI media: Geophysics, 77, no. 4, C37-C42, doi: 10.1190/geo2011-0158.1.

Tao, G., and M. S. King, 1990, Shear-wave velocity and Q anisotropy in rocks: A laboratory study: International Journal of Rock Mechanics and Mining Sciences and Geomechanics Abstracts, 27, 353-361, doi: $10.1016 / 0148-9062(90) 92710-\mathrm{V}$.

Thomsen, L., 1986, Weak elastic anisotropy: Geophysics, 51, 1954-1966, doi: $10.1190 / 1.1442051$

Thomson, C. J., 1997, Complex rays and wave packets for decaying signals in inhomogeneous, anisotropic and anelastic media: Studia Geophysica et Geodaetica, 41, 345-381, doi: 10.1023/A:1023359401107.

Tsvankin, I., 1997, Anisotropic parameters and P-wave velocity for orthorhombic media: Geophysics, 62, 1292-1309, doi: 10.1190/1.1444231.

van Trier, J., and W. W. Symes, 1991, Upwind finite-difference calculation of traveltimes: Geophysics, 56, 812-821, doi: 10.1190/1.1443099.

Vavryčuk, V., 2006, Calculation of the slowness vector from the ray vector in anisotropic media: Proceedings of the Royal Society, Series A, 462, 883896, doi: 10.1098/rspa.2005.1605.

Vavryčuk, V., 2007, Ray velocity and ray attenuation in homogeneous anisotropic viscoelastic media: Geophysics, 72, no. 6, D119-D127, doi: 10 $.1190 / 1.2768402$

Vavryčuk, V., 2008, Real ray tracings in anisotropic viscoelastic media: Geophysical Journal International, 175, 617-626, doi: 10.1111/j.1365-246X .2008.03898.x.

Vavryčuk, V., 2009, Weak anisotropy-attenuation parameters: Geophysics, 74, no. 5, WB203-WB213, doi: 10.1190/1.3173154.

Vavryčuk, V., 2010, Behaviour of rays at interfaces in anisotropic viscoelastic media: Geophysical Journal International, 181, 1665-1677.

Vavryčuk, V., 2012, On numerically solving the complex eikonal equation using real ray-tracing methods: A comparison with the exact analytical solution: Geophysics, 77, no. 4, T109-T116, doi: 10.1190/geo2011-0431.1.

Vidale, J. E., 1988, Finite-difference calculation of travel times: Bulletin of the Seismological Society of America, 78, 2062-2076.

Vidale, J. E., 1990, Finite-difference calculation of traveltimes in three dimensions: Geophysics, 55, 521-526, doi: 10.1190/1.1442863.

Zhang, Y.-T., H.-K. Zhao, and J. Qian, 2006, High order fast sweeping methods for static Hamilton-Jacobi equations: Journal of Scientific Computing, 29, 25-56, doi: 10.1007/s10915-005-9014-3.

Zhu, T. F., and K. Y. Chun, 1994, Complex rays in elastic and anelastic media: Geophysical Journal International, 119, 269-276, doi: 10.1111/ j.1365-246X.1994.tb00927.x.

Zhu, Y., and I. Tsvankin, 2006, Plane-wave propagation in attenuative transversely isotropic media: Geophysics, 71, no. 2, T17-T30, doi: 10.1190/1 .2187792 .

Zhu, Y., and I. Tsvankin, 2007, Plane-wave attenuation anisotropy in orthorhombic media: Geophysics, 72, no. 1, D9-D19, doi: 10.1190/1.2387137.

Zhubayev, A., M. E. Houben, D. M. J. Smeulders, and A. Barnhoorn, 2016, Ultrasonic velocity and attenuation anisotropy of shales, Whitby, United Kingdom: Geophysics, 81, no. 1, D45-D56, doi: 10.1190/geo2015-0211.1. 